CLINICAL STUDY

\title{
Correlates of increased lean muscle mass in women with polycystic ovary syndrome
}

\author{
E Carmina $^{1}$, E Guastella $^{2}$, R A Longo $^{1}$, G B Rini $^{1}$ and R A Lobo ${ }^{3}$ \\ Departments of ${ }^{1}$ Clinical Medicine and ${ }^{2}$ Obstetrics and Gynecology, University of Palermo, Palermo, Italy and ${ }^{3}$ Department of Obstetrics and Gynecology, \\ Columbia University Medical Center, Columbia University, 622 West, 168th Street, New York, New York 10032, USA \\ (Correspondence should be addressed to R A Lobo; Email: ral35@columbia.edu)
}

\begin{abstract}
Objective: Muscle mass plays an important role in determining cardiovascular and metabolic risks in polycystic ovary syndrome (PCOS). In addition, whether lean mass influences carotid intima-media thickness (IMT) in PCOS has not been assessed.

Design: Prospective investigation.

Methods: Ninety-five women with PCOS were age- and weight-matched to 90 ovulatory controls. All women had dual X-ray absorptiometry for lean, fat and bone mass, and bone mass density (BMD). Serum testosterone, sex hormone-binding globulin, insulin, and glucose and carotid IMT were determined. Free androgen index (FAI) and insulin resistance (by QUICKI) were calculated.

Results: In PCOS, waist circumference and insulin were higher and QUICKI lower than in controls $(P<0.01)$. Trunk fat mass, \% trunk fat, and lean mass were higher in PCOS compared to controls $(P<0.01)$, while total bone mass and BMD were similar. IMT was increased in PCOS $(P<0.01)$ but only $15 \%$ of PCOS patients had abnormal $(\geq 0.9 \mathrm{~mm})$ values. Lean mass correlated with fat parameters, insulin, QUICKI, and FAI, but not with total testosterone; and after adjustments for insulin and QUICKI, lean mass still correlated with fat mass $(P<0.01)$ but not FAI. Lean mass correlated with IMT $(P<0.01)$, but this was dependent on insulin. However, excluding those patients with abnormal IMT values, IMT correlated with lean mass independently of insulin. Bone mass correlated with lean and fat mass, but not with insulin or androgen. PCOS patients with 'pathological' IMT values had higher \% trunk fat, lean mass, and insulin, lower QUICKI, and higher testosterone and FAI compared with those with normal IMT.

Conclusion: Lean mass is increased in PCOS, while bone mass is similar to that of matched controls. The major correlates of lean mass are fat mass and insulin but not androgen. Lean mass also correlated with IMT, and although influenced by insulin, small changes in IMT may partially reflect changes in muscle mass, while clearly abnormal values relate to more severe abnormalities of PCOS.
\end{abstract}

European Journal of Endocrinology 161 583-589

\section{Introduction}

In recent years, many studies have focused on fat quantity and distribution in women with polycystic ovary syndrome (PCOS) (1-4). These and other studies have shown that increased abdominal fat is an important determinant of increased cardiovascular and metabolic disease $(4,5)$. Most studies have shown that increased abdominal fat is present not only in the majority of women with PCOS who are obese but also occurs in overweight and normoweight women with the syndrome $(1,2)$.

However, there is a paucity of data on other components of body structure and their possible effects on cardiovascular risk in PCOS. In particular, data on lean muscle mass in PCOS are few and contradictory.
It has been shown that obese women also tend to have increased lean mass (6); this trend may be higher in women with PCOS where other factors such as obesity, insulin resistance, and androgen excess may also contribute to increasing lean mass. In fact, initial studies evaluating lean mass in Japanese women with PCOS have suggested that there is some degree of increased muscle mass in PCOS (6), which was considered to be related to the androgen excess of these women $(7,8)$.

On the contrary, in another study, lean mass was reported to be reduced (9). In that study, body composition was measured only in a small group $(n=10)$ of lean women with PCOS. Finally, lean mass was found to be decreased in another small $(n=22)$ study of hyperandrogenic insulin-resistant young 
women (aged 17-21 years), who were selected on the basis of having a normal body mass index (BMI) (10).

The studies cited above did not critically assess lean mass in the typical population of women with PCOS who are predominantly overweight or obese $(11,12)$. Since lean muscle mass contributes significantly to metabolism, and represents one of the major targets of both insulin and androgen action (13), this determinant may be an important parameter of body composition to assess in PCOS.

The importance of determining muscle mass in PCOS has been further increased by the finding that changes in lean mass appear to correlate with changes in some cardiovascular parameters, including a correlation between small increases in carotid intima-media thickness (IMT) with larger muscle mass (14, 15). This finding opens the possibility that some of the cardiovascular changes that have been found in PCOS (16), may simply be related to changes in lean muscle mass as well. In particular, carotid IMT has been found to be increased even in young women with PCOS $(17,18)$ and is generally believed to reflect the early appearance of atherosclerosis (19). However, in most women with PCOS, the increase in carotid IMT is generally small and rapidly reversible with metformin (20); a finding that is uncommon in the general population (21).

In this study, we measured lean muscle mass and carotid IMT in a large population of women with PCOS and matched controls. To evaluate lean mass, we used total body dual X-ray absorptiometry (DEXA), a methodology that permits a good assessment of the main components of body composition, including fat and lean mass (22-24). We also evaluated the possible correlations between the lean mass and carotid IMT and assessed the possible influences of insulin and androgens.

\section{Materials and methods}

\section{Subjects}

Ninety-five women with PCOS (mean age 24.2 \pm 3 years, range 18-40 years, and a mean BMI of $2 \overline{7} .6$ $\pm 5.8)$ were evaluated consecutively; a total of 33 patients were obese $(\mathrm{BMI} \geq 30), 31$ were overweight (BMI 25-29.9), and 31 had normal weight (BMI 19-24.9). These women had been referred to the Endocrine Unit of the Department of Clinical Medicine of the University of Palermo because of symptoms of hyperandrogenism and were found to be affected by PCOS. The diagnosis of PCOS was based on the criteria of hyperandrogenism (clinical or biochemical) plus chronic anovulation and/or polycystic ovaries on ultrasound with the exclusion of adrenal enzymatic deficiencies, Cushing's syndrome, and tumors (25). Clinical hyperandrogenism was defined by the presence of hirsutism. Hirsutism was assessed by FerrimanGallwey-Lorenzo scores with patients with scores $\geq 8$ being considered as hirsute. Biochemical hyperandrogenism was defined as finding elevated levels of serum androgens (testosterone and/or free androgen index (FAI), and/or DHEA sulfate, which were above the 95\% confidence intervals of a normal ovulatory population of women of similar age) (26). Anovulation was defined as serum $\mathrm{P}<3 \mathrm{ng} / \mathrm{ml}$. In patients with normal menses, at least two consecutive menstrual cycles were studied, and the finding of low levels of serum $\mathrm{P}(<3 \mathrm{ng} / \mathrm{ml})$ in both cycles was considered as being consistent with the presence of chronic anovulation. Normal circulating levels of 17-hydroxyprogesterone $(<2 \mathrm{ng} / \mathrm{ml})$ were used to exclude the diagnosis of nonclassical 21-hydroxylase deficiency (26), while clinical data and/or urinary cortisol assessment were utilized to exclude the diagnosis of Cushing's syndrome. Patients with androgen secreting tumors were excluded by not finding very high androgen levels $(>2.5$ times the upper normal range) or the demonstration of tumors by computed tomography (CT) scan or magnetic resonance imaging.

Ninety age- and weight-matched normal ovulatory women (aged 18-40 years, mean age 23.9 \pm 3 years, and mean BMI 27.5 \pm 3 ) were also studied and served as controls. The controls were selected on the basis of having no hirsutism or biochemical hyperandrogenism and normal ovulatory menstrual cycles, and were matched for age and body weight with the patients with PCOS. These healthy women were recruited from family members of hospital co-workers of the Department of Clinical Medicine of the University of Palermo. The presence of normal ovulation was assessed by the measurement of serum progesterone on days 22-23 of the cycle, when progesterone was tested and was $>7 \mathrm{ng} / \mathrm{ml}$.

The main objective of the study design was to match patients and controls not only by body weight, but also by body weight distribution so as to have a similar number of controls and women with PCOS in the subgroups of normoweight (BMI 20-24.9), overweight (BMI 25-29.9), and obese (BMI 30-49). Since we have found that in our population of women with PCOS in Palermo, Italy, these three different weight subgroups are equally represented (12), we planned to study at least 30 patients and 30 controls in each weight subgroup. In order to accomplish this, we consecutively included patients who had PCOS and divided them into these three subgroups based on BMI. For every ten women with PCOS in a particular weight subgroup who were studied, ten controls were selected and matched based upon body characteristics. The final number was slightly higher than the 30 anticipated in the overweight subgroup, and this subgroup was slightly larger. In all patients and controls, height, weight, and waist circumference (measured at the midpoint between the lateral iliac crest and the lowest rib margin at the end of 
normal expiration) were recorded and BMI was calculated in $\mathrm{kg} / \mathrm{m}^{2}$.

The design of the study included assessment of body composition, evaluation of carotid IMT, and measurements in fasting blood of testosterone, sex hormonebinding globulin (SHBG), insulin, and glucose.

\section{Evaluation of body composition}

In all patients and controls, body composition was determined by total body DEXA using a QDR Discovery Hologic instrument (2). The following parameters were measured: total lean mass $(\mathrm{g})$, total fat mass $(\mathrm{g})$, trunk fat mass (g) and \% trunk fat mass, total bone mass (g), and bone mass density (BMD). Because total lean mass is highly dependent on height, lean mass was expressed as total lean mass/height in $\mathrm{cm}$.

The intra-coefficient of variation (CV) for repeated measures of body composition parameters was $<3 \%$.

\section{Carotid IMT assessment}

All women were examined in the supine position, with the head hyper-extended and turned away from the side being scanned. Scans were performed by an experienced ultrasonographer ( $\mathrm{R}$ A L, Italy), blinded to clinical data, using a color Doppler (Vingmed System Five, GE, Horten, Norway) with a high resolution 10-MHz linear probe. Sonographic scans were obtained of the right and left common carotid artery and the carotid bifurcation bulb area from multiple planes (lateral, anterior oblique, and posterior oblique). Images were obtained from the distal portion of both common carotid arteries, 1-2 cm proximal to the carotid bulb, immediately proximal to the origin of the bifurcation. To simplify the scanning procedure, and because of the lesser stability of measurements of the internal and external carotid artery, these segments were not taken into consideration in the present study $(19,27)$. The IMT of the posterior (far) wall of both common carotid arteries was measured as the distance between the junction of the lumen and intima and that of the media and adventitia $(27,28)$.

Carotid IMT was measured during end-diastole from the B-mode screen. The mean IMT for each carotid was calculated as the average of ten measurements made in the right and left carotid arteries using electronic calipers. Ambient light and temperature were controlled throughout the procedure. The intra-CV for the repeated measurements of IMT was $<7.0 \%$.

Because carotid IMT is influenced by age, race, and sex (29) and very few data exist on normal and 'pathologic' IMT values in the young female population, values higher than the upper 95th percentile in our normal control population were considered to be pathologic $(29,30)$. The threshold value for abnormally high IMT was found to be $\geq 0.90 \mathrm{~mm}$, which is similar to that found in other studies when a relatively young population was studied $(29,31)$, and is lower than that reported in studies involving males or older subjects $(\geq 1.00 \mathrm{~mm})(29,32)$.

\section{Assays}

Between 0800 and $0900 \mathrm{~h}$ during the follicular phase (days 5-8), fasting blood samples for measurement of testosterone, SHBG, insulin, and glucose were obtained. FAI (33) and insulin resistance (by assessment of QUICKI) (34) were calculated.

Serum testosterone was measured by RIA after extraction and chromatography (35). SHBG was measured by a RIA method using materials provided by Diagnostic Systems Laboratories, Inc. (Webster, TX, USA). Plasma glucose levels were determined by the glucose oxidase technique. Insulin was determined with a double antibody method using reagents obtained from Linco Research, Inc. (St Charles, MO, USA).

In all hormonal assays, the intra-assay CV was $<6 \%$, and the interassay CV was $<15 \%$.

Institutional review board approval was obtained, and all patients and controls gave written consent. All subjects were considered to be sedentary and were not dieting or receiving any medications. No subject received hormonal medications for at least 3 months before the study.

\section{Statistical analyses}

ANOVA was used for comparisons. Post hoc testing was carried out by Student's $t$-test with log transformation. Analysis of covariance was used to assess the role of insulin, insulin resistance, and androgens on correlations between body components and IMT. Pearson product moment correlation and stepwise multivariate linear regression analysis with forward selection were used to analyze correlations. $P<0.05$ was considered statistically significant. All data are expressed as mean \pm s.D.

\section{Results}

Women with PCOS had increased $(P<0.01)$ waist circumference, testosterone, FAI, and insulin values and lower $(P<0.01)$ levels of QUICKI when compared with controls (Table 1).

Table 1 Clinical and hormonal data in 95 women with polycystic ovary syndrome (PCOS) and 90 weight-matched controls.

\begin{tabular}{lcc}
\hline & Controls & PCOS \\
\hline BMI $\left(\mathrm{kg} / \mathrm{m}^{2}\right)$ & $26.8 \pm 3$ & $27.5 \pm 5.8$ \\
Waist circumference $(\mathrm{cm})$ & $85.4 \pm 7$ & $92.7 \pm 13^{\dagger}$ \\
Testosterone $(\mathrm{ng} / \mathrm{dl})$ & $35 \pm 13$ & $72.2 \pm 33^{*}$ \\
FAI & $2.5 \pm 0.8$ & $8.72 \pm 5.1^{\dagger}$ \\
Insulin $(\mu \mathrm{U} / \mathrm{ml})$ & $9.6 \pm 3$ & $14 \pm 5.2^{\dagger}$ \\
QUICKI & $0.348 \pm 0.02$ & $0.328 \pm 0.02^{\dagger}$ \\
\hline
\end{tabular}

${ }^{\star} P<0.05$ versus controls; ${ }^{\dagger} P<0.01$ versus controls. 
Table 2 Body composition in 95 women with polycystic ovary syndrome (PCOS) and 90 weight-matched ovulatory controls.

\begin{tabular}{llllll}
\hline & Total fat $(\mathrm{g})$ & Trunk fat $(\mathrm{g})$ & $\%$ Trunk fat & $\begin{array}{l}\text { Lean mass } \\
\text { (total g/height cm) }\end{array}$ & $\begin{array}{l}\text { Bone mass }(\mathrm{g}) \\
\text { Bensity }\left(\mathrm{g} / \mathrm{cm}^{2}\right)\end{array}$ \\
\hline Controls & $25542 \pm 5705$ & $8882 \pm 3120$ & $34.8 \pm 4$ & $256 \pm 27$ & $2119 \pm 273$ \\
PCOS & $25734 \pm 9658$ & $10708 \pm 4320$ & $41.6 \pm 6$ & $275 \pm 38$ & $21.09 \pm 0.08$ \\
& & $P<0.01$ & $P<0.01$ & $P<0.01$ & $1.10 \pm 0.07$ \\
\hline
\end{tabular}

Analysis of body composition demonstrated that women with PCOS and matched controls had similar total fat quantity. However, women with PCOS had significantly $(P<0.01)$ higher values of trunk fat, and \% trunk fat in comparison with weight-matched controls (Table 2). Lean mass was significantly higher in women with PCOS compared with the controls (Table 2). Total bone mass was similar in the groups and was remarkably close in values, each averaging $\sim 2100 \mathrm{~g}$ (Table 2). BMD was also almost identical in PCOS and controls (Table 2).

Dividing women with PCOS on the basis of BMI, it was found that lean and overweight women with PCOS had slightly but not significantly higher lean mass compared with controls of similar BMI; obese women with PCOS had significantly higher lean mass compared with obese controls $(331 \pm 32$ vs 278 $\pm 28 \mathrm{~g} / \mathrm{cm}, P<0.01)$.

Women with PCOS had significantly $(P<0.01)$ increased IMT $(0.61 \pm 0.18 \mathrm{~mm})$ in comparison with both weight-matched controls $(0.53 \pm 0.15 \mathrm{~mm})$. Fifteen women with PCOS (16\%) had values of IMT higher than $0.90 \mathrm{~mm}$ (representing the upper 95\% confidence intervals of values in matched normal women derived from previous data (18)), while none of the controls in this study were found to have abnormally increased IMT.

\section{Analyses of women with pathologic IMT values}

The 15 women with IMT values $\geq 0.90 \mathrm{~mm}$ (mean IMT: $1 \pm 0.1 \mathrm{~mm}$ ), compared with the other women with PCOS were of similar age ( $25 \pm 6$ vs $24 \pm 3$ years) and had similar BMI and waist circumference but significantly higher basal insulin, lower QUICKI, and higher testosterone and FAI (Table 3). Analysis of body composition showed that women with pathologic IMT values had similar total body fat, but significantly higher \% trunk fat and lean mass/cm (Table 4). Three women with pathologic IMT values were lean with normal total fat, but also higher \% trunk fat than is considered normal ( $>40 \%)$.

\section{Correlations}

The correlations of lean mass with body fat parameters and endocrine variables (PCOS subjects and controls) are depicted in Table 5. Lean mass significantly
$(P<0.01)$ correlated with BMI, waist circumference, total and trunk fat mass, insulin, QUICKI, and FAI, but not with total testosterone. After adjustments for serum insulin or QUICKI, the lean mass correlation with total and trunk fat remained significant $(P<0.01)$, while the correlation with FAI disappeared.

Lean mass also correlated with IMT $(r 0.36$, $P<0.01)$. As depicted in Table 6, IMT significantly $(P<0.01)$ correlated with serum insulin and QUICKI, but not with fat parameters except for $\%$ trunk fat. IMT did not correlate with serum testosterone or FAI. After correction for serum insulin, the correlation of lean mass with IMT disappeared. However, when patients with abnormal values of IMT $(>0.9 \mathrm{~mm})$ were excluded, the correlation of lean mass and IMT remained significant $(P<0.05)$ even after adjusting for insulin.

Total bone mass correlated significantly with lean body mass $(r=0.43, P<0.01)$ and with total fat mass $(r=0.29, P<0.05)$, but not with other parameters including insulin and testosterone.

When patients and controls were evaluated separately, in both groups lean mass correlated with BMI, waist circumference, total and trunk fat mass, insulin, QUICKI, and FAI, but not with total testosterone. In women with PCOS, IMT correlated with serum insulin, QUICKI, trunk fat, \% trunk fat and lean mass, while in controls IMT correlated only with insulin and QUICKI.

Table 3 Clinical and hormonal data in 15 polycystic ovary syndrome (PCOS) patients with pathologic intima-media thickness (IMT; $\geq 0.9 \mathrm{~mm}$ ) and in 80 PCOS patients with normal IMT $(<0.9 \mathrm{~mm})$.

\begin{tabular}{lcc}
\hline & $\begin{array}{c}\text { Patients with } \\
\text { IMT in normal } \\
\text { range }\end{array}$ & $\begin{array}{c}\text { Patients with } \\
\text { pathologic IMT }\end{array}$ \\
\hline Age & $24 \pm 2$ & $25 \pm 6$ \\
BMI & $26.9 \pm 5.5$ & $30.5 \pm 8$ \\
Waist circumference (cm) & $92 \pm 13$ & $96.7 \pm 14.9$ \\
Total testosterone $(\mathrm{ng} / \mathrm{dl})$ & $69 \pm 34$ & $88 \pm 32^{*}$ \\
FAl & $7.7 \pm 6$ & $14.6 \pm 9.8^{\dagger}$ \\
Insulin $(\mu \mathrm{U} / \mathrm{ml})$ & $12.7 \pm 5$ & $21.2 \pm 7.7^{\dagger}$ \\
QUICKI & $0.332 \pm 0.02$ & $0.308 \pm 0.018^{\dagger}$
\end{tabular}

${ }^{\star} P<0.05$ versus patients with normal IMT; ${ }^{\dagger} P<0.01$ versus patients with normal IMT. 
Table 4 Body composition in 80 polycystic ovary syndrome (PCOS) patients with normal intima-media thickness (IMT; $<0.9 \mathrm{~mm})$ and $15 \mathrm{PCOS}$ patients with pathologic IMT $(\geq 0.9 \mathrm{~mm})$.

\begin{tabular}{lcc}
\hline & Normal IMT & Pathologic IMT \\
\hline Total fat $(\mathrm{g})$ & $24996 \pm 10100$ & $32336 \pm 14165$ \\
Trunk fat $(\mathrm{g})$ & $10038 \pm 4900$ & $14283 \pm 6816^{*}$ \\
$\%$ Trunk fat & $41.2 \pm 4$ & $43.7 \pm 2.6^{\dagger}$ \\
Lean mass/cm & $264 \pm 50$ & $335 \pm 105^{\star}$ \\
\hline
\end{tabular}

${ }^{\star} P<0.05$ versus patients with normal IMT; ${ }^{\dagger} P<0.01$ versus patients with normal IMT.

\section{Discussion}

Our study has demonstrated that lean muscle mass is increased in women with PCOS compared with weightmatched controls. While in the past contrasting data have been reported, all previous studies determined the quantity of lean mass in lean women with PCOS (7-9). On the contrary, here we evaluated the most common phenotype of PCOS, namely women who were overweight or obese (mean $\mathrm{BMI}=28$ ) and found that lean mass was higher in PCOS than in age- and weightmatched controls. To be sure that the differences were not influenced by body height, the lean mass was expressed as total lean mass/height in $\mathrm{cm}$ but this, also, was significantly higher in PCOS compared with controls.

In our study, lean mass was highly correlated with altered fat parameters and to insulin and FAI but not testosterone. The correlations of fat and insulin with lean mass were partially independent of each other, suggesting that not only insulin but perhaps other factors in adipose tissue may play a role in increasing lean mass. Nevertheless, our study suggests that testosterone has a limited effect on lean muscle mass, at least in terms of total testosterone, and the correlation with FAI is likely determined by lower SHBG and elevated insulin. Accordingly, previous studies, which did not find increased lean mass in PCOS, could be explained by the study of only lean women with PCOS having little or no insulin resistance $(7,9)$. The only study that evaluated lean mass in hyperinsulinemic women assessed a particular population of very young hyperandrogenic lean women (10). Consistent with our data, Glintborg has recently

Table 5 Correlation coefficients $(r)$ of lean mass (total lean mass in $\mathrm{g} /$ height in $\mathrm{cm}$ ) with fat mass and endocrine parameters.

\begin{tabular}{ll}
\hline & Lean mass \\
\hline Total fat & $0.68(P<0.01)$ \\
Trunk fat & $0.67(P<0.01)$ \\
$\%$ Trunk fat & $0.48(P<0.01)$ \\
Testosterone & 0.11 \\
FAI & $0.48(P<0.01)$ \\
Insulin & $0.49(P<0.01)$ \\
QUICKI & $-0.54(P<0.01)$ \\
\hline
\end{tabular}

reported that in women with PCOS, lean mass is reduced by pioglitazone, an insulin-sensitizing drug that decreases insulin levels (36).

Finding an increased lean mass in women with PCOS may be important because muscle is one of the main targets for the effects of insulin, and metabolic effects of insulin and insulin resistance are partially dependent on the quantity of muscle mass (13). The increase in lean mass may also influence the cardiovascular phenotype of the patients. It has been shown that in the general population, small increases in the carotid IMT, a factor that is generally considered a sign of vascular damage and atherosclerosis, may be merely the consequence of an adaption of the cardiovascular system to an increased lean mass $(14,16)$. In fact, changes in cardiac output are seen in these conditions which in turn may be associated with small increases in IMT $(14,15)$. Similar correlations were found between total and trunk fat and IMT suggesting that different body constituents (both fat and lean mass) may have a role in determining increased IMT. Clearly, we are aware that merely finding a correlation does not necessarily mean that there is a causal relationship; prospective investigations will be necessary. However, our study does suggest a possible role for different body constituents in modifying carotid IMT.

In PCOS, several studies have shown increased carotid IMT $(17,18)$ and have been considered to be a sign of the early development of atherosclerosis in these women. However, current evidence suggests that cardiovascular events in PCOS are only slightly increased (37-39) and there is a discrepancy between the common finding of increased markers in early atherosclerosis and the development of cardiovascular events. In addition, it has been shown that increased IMT in young women with PCOS may be rapidly reversible with metformin (20), a finding that is different from that found in the general population (21).

Our study showing a correlation between increased lean and fat mass and increased carotid IMT, suggests, therefore, that changes in body composition may be at least partially responsible for the observed increase in carotid IMT in PCOS. It is important to note that this is not to refute the notion that some women with PCOS

Table 6 Correlations coefficients $(r)$ of intima-media thickness (IMT; $\mathrm{mm}$ ) with parameters of body composition and endocrine values.

\begin{tabular}{lc}
\hline & IMT \\
\hline Total fat & 0.19 \\
Trunk fat & 0.20 \\
\% Trunk fat & $0.35^{*}$ \\
Lean mass & $0.31^{*}$ \\
Testosterone & 0.19 \\
FAI & 0.20 \\
Insulin & $0.42^{*}$ \\
QUICKI & $-0.46^{*}$ \\
\hline
\end{tabular}

${ }^{\star} P<0.01$. 
may have significant atherosclerosis. We suggest that in many, perhaps most, young women with PCOS, the observed small increases in IMT are at least partially dependent on paraphysiologic changes linked to increased lean muscle mass. Some patients, however, may have more severe findings. In fact, on evaluating a large group of patients, we found that $16 \%$ of young patients with PCOS have abnormal or pathologic $(\geq 0.90 \mathrm{~mm})$ values of IMT; this finding suggests atherosclerotic changes that may need more assessment. Previous studies did not report pathologic changes of IMT but only indicated a mean increase in carotid IMT, well within the normal range.

An important finding in this study is that women with PCOS who have pathologic IMT values have more significant abdominal obesity and more severe abnormalities related to PCOS. Also lean mass was higher in women with PCOS who had pathologic IMT values, suggesting that in these women body composition may be modified by more severe insulin resistance and androgen excess. These women were more commonly found to be obese (although three were not) and we found an increase in \% trunk or abdominal fat in all these women.

More data are needed, but the data at hand suggest that in young women with PCOS, a clear distinction should be made in the findings of increased IMT. In the majority of women, this finding may be an adaptive change, while in others it may have a more ominous connotation. Our data suggest that the finding of abdominal obesity ( $\%$ trunk fat of $>40 \%$ ) may be a marker of this abnormality.

In conclusion, our data suggest that patients with PCOS have an increase in lean mass that is highly associated with hyperinsulinemia and altered fat parameters, and less so with androgen; it may be correlated with some changes in carotid IMT, which may not have a particularly significant pathological consequence. However, in a minority of women with PCOS, $16 \%$ in this study, abnormal or pathological increases in IMT may be found. This group of women with significant increases in carotid IMT measurements may require more rigorous assessment and possible treatment.

\section{Declaration of interest}

No conflict of interest that could be perceived.

\section{Funding}

This research did not receive any specific grant from any funding agency in the public, commercial, or not-for-profit sector.

\section{References}

1 Puder JJ, Varga S, Kraenzlin M, De Geyter C, Keller U \& Muller B. Central fat excess in polycystic ovary syndrome: relation to low grade inflammation and insulin resistance. Journal of Clinical Endocrinology and Metabolism 200590 6014-6021.
2 Carmina E, Bucchieri S, Esposito A, Del Puente A, Mansueto P, Di Fede G \& Rini GB. Abdominal fat quantity and distribution in women with polycystic ovary syndrome and extent of its relation to insulin resistance. Journal of Clinical Endocrinology and Metabolism 200792 2500-2505.

3 Barber TM, Golding SJ, Alvey C, Wass JA, Karpe F, Franks S \& McCarthy MI. Global adiposity rather than abnormal regional fat distribution characterizes women with polycystic ovary syndrome. Journal of Clinical Endocrinology and Metabolism $2008 \mathbf{9 3}$ 999-1004.

4 Lord J, Thomas R, Fox B, Acharya U \& Wilkin T. The central issue? Visceral fat is a good marker of insulin resistance and metabolic disturbances in women with polycystic ovary syndrome British Journal of Obstetrics and Gynaecology $2006 \mathbf{1 1 3}$ 1203-1209.

5 Karelis AD, St-Pierre DH, Conus F, Rabasa-Lhoret R \& Poehlman ET. Metabolic and body composition factors in subgroups of obesity: what do we know? Journal of Clinical Endocrinology and Metabolism 200489 2569-2575.

6 Forbes GB. Longitudinal changes in adult fat-free mass: influence of body weight. American Journal of Clinical Nutrition $1999 \mathbf{7 0}$ 1025-1031.

7 Douchi T, Oki T, Yamasaki H, Kuwahata R, Nakae M \& Nagata Y. Relationship of androgens to muscle size and bone mineral density in women with polycystic ovary syndrome. Obstetrics and Gynecology 200198 445-449.

8 Notelovitz M. Androgen effects on bone and muscle. Fertility and Sterility $20024534-541$.

9 Kirchengaast S \& Huber J. Body composition characteristics and body fat distribution in lean women with polycystic ovary syndrome. Human Reproduction 200116 1255-1260.

10 Ibanez L \& De Zegher F. Ethynilestradiol-drospirenone, flutamidemetformin or both for adolescents and women with hyperinsulinemic hyperandrogenism: opposite effects on adipocytokines and body adiposity. Journal of Clinical Endocrinology and Metabolism $2004891592-1597$.

11 Carmina E, Legro RS, Stamets K, Lowell J \& Lobo RA. Difference in body weight between American and Italian women with polycystic ovary syndrome: influence of the diet. Human Reproduction $2003112289-2293$.

12 Essah P, Nestler JE \& Carmina E. Differences in dyslipidemia between American and Italian women with polycystic ovary syndrome. Journal of Endocrinological Investigation 200831 35-41.

13 Corbould A, Zhao H, Mirzoeva S, Aird D \& Dunaif A. Enhanced mitogenic signaling in skeletal muscle in women with polycystic ovary syndrome. Diabetes $2006 \mathbf{5 1} 751-759$.

14 Bots ML, Hofman A \& Grobbee DE. Increased common carotid intima-media thickness. Adaptive response or a reflection of atherosclerosis? Findings from the Rotterdam study. Stroke 1997 28 2442-2447.

15 Kozakowa M, Palombo C, Paterni M, Anderwald CH, Konrad T, Colgan MP, Flyvbjerg A \& Dekker J. Body composition and common carotid artery remodeling in a healthy population. Journal of Clinical Endocrinology and Metabolism 200893 3325-3332.

16 Orio F Jr, Palomba S, Spinelli L, Cascella T, Tauchmanova L, Zullo F, Lombardi G \& Colao A. The cardiovascular risk of young women with polycystic ovary syndrome: an observational, analytical, prospective case-control study. Journal of Clinical Endocrinology and Metabolism 200489 3696-3701.

17 Orio F Jr, Palomba S, Cascella T, De Simone B, Di Biase S, Russo T, Labella D, Zullo F, Lombardi G \& Colao A. Early impairment of endothelial structure and function in young normal-weight women with polycystic ovary syndrome. Journal of Clinical Endocrinology and Metabolism 200489 4588-4593.

18 Carmina E, Orio F, Palomba S, Longo RA, Cascella T, Colao A, Lombardi G, Rini GB \& Lobo A. Endothelial dysfunction in PCOS: role of obesity and adipose hormones. American Journal of Medicine 2006119 356.e1-356.e6. 
19 Crouse JR III, Craven TE, Hagaman AP \& Bond MG. Association of coronary disease with segment-specific intimal-medial thickening of the extracranial carotid artery. Circulation 199592 1141-1147.

20 Orio F Jr, Palomba S, Cascella T, De Simone B, Manguso F Savastano S, Russo T, Tolino A, Zullo F, Lombardi G, Azziz R \& Colao A. Improvement in endothelial structure and function after metformin treatment in young normal-weight women with polycystic ovary syndrome: results of a 6-month study. Journal of Clinical Endocrinology and Metabolism $2005906072-6076$.

21 Ubels FL, Terpstra WF \& Smith AJ. Carotid intima-media thickness: influence of treatment and clinical implications. Netherlands Journal of Medicine 199955 188-195.

22 Strauss BJ, Gibson PR, Stroud DB, Borovnicar DJ, Xiong DW \& Keogh J. Total body dual X-ray absorptiometry is a good measure of both fat mass and fat free mass in liver cirrhosis compared to "gold standard" techniques. Melbourne liver group. Annals of the New York Academy of Sciences 2000904 55-62.

23 Miller A, Strauss BJ, Mol S, Kyoonq A, Holmes PH, Finlay P, Bardin PG \& Guy P. Dual energy X-ray absorptiometry is the method of choice to assess body composition in COPD. Radiology $200914411-418$.

24 Andreoli A, Scalzo G, Masala S, Tarantino U \& Guglielmi G. Body composition assessment by dual energy X-ray absorptiometry. $\mathrm{La}$ Radiologia Medica 2009114 286-300.

25 Azziz R, Carmina E, Dewailly D, Diamanti-Kandarakis E, EscobarMorreale HF, Futterweit W, Janssen OE, Legro RS, Norman RJ, Taylor AE \& Witchel SF. Position statement: criteria for defining polycystic ovary syndrome as a predominantly hyperandrogenic syndrome: an androgen excess society guideline. Journal of Clinical Endocrinology and Metabolism 200691 4237-4245.

26 Carmina E, Rosato F, Jannì A, Rizzo M \& Longo RA. Relative prevalence of different androgen excess disorders in 950 women referred because of clinical hyperandrogenism. Journal of Clinical Endocrinology and Metabolism 200691 2-6.

27 Salonen JT \& Salonen R. Ultrasonographically assessed carotid morphology and the risk of coronary heart disease. Arteriosclerosis, Thrombosis, and Vascular Biology 199111 1245-1249.

28 Gamble G, Beaumont B, Smith H, Zorn J, Sanders G, Merrilees J, MacMahon S \& Sharpe N. B-mode ultrasound images of the carotid artery wall: correlation of ultrasound with histological measurements. Atherosclerosis 1993102 163-173.

29 Roman MJ, Naqvi TZ, Gardin JM, Gerhard-Herman M, Jaff M \& Mohler E. American society of echocardiography report. Clinical application of noninvasive vascular ultrasound in cardiovascular risk stratification: a report from the American Society of Echocardiography and the Society for Vascular Medicine and Biology. Vascular Medicine 200611 201-211.
30 Simon A, Chironi J \& Levenson J. Comparative performance of subclinical atherosclerosis tests in predicting coronary heart disease in asymptomatic individuals. European Heart Journal 200728 2967-2971.

31 Bots ML, Hoes AW, Koudstaal PJ, Hofman A \& Grobbee DE. Common carotid intima-media thickness and risk of stroke and myocardial infarction: the Rotterdam study. Circulation 199796 1432-1437.

32 Howard G, Sharrett AR, Heiss G, Evans GW, Chambless LE, Riley WA \& Burke GL. Carotid artery intimal-medial thickness distribution in general populations as evaluated by B-mode ultrasound. ARIC Investigators. Stroke 199324 1297-1304.

33 Morley JE, Patrick P \& Perry HM III. Evaluation of assays available to measure free testosterone. Metabolism 200251 554-559.

34 Katz A, Nambi SS, Mather K, Baron AD, Follmann DA, Sullivan G \& Quon MJ. Quantitative insulin sensitivity check index: a simple, accurate method of assessing insulin sensitivity in humans. Journal of Clinical Endocrinology and Metabolism $2000 \mathbf{8 5}$ 2402-2410.

35 Carmina E. Prevalence of idiopathic hirsutism. European Journal of Endocrinology $1998139424-427$.

36 Glintborg D, Andersen M, Hagen C, Heickendorff L \& Hermann AP. Association of pioglitazone treatment with decreased bone mineral density in obese premenopausal patients with polycystic ovary syndrome: a randomized, placebo controlled trial. Journal of Clinical Endocrinology and Metabolism 200893 1696-1701.

37 Pierpoint T, McKeigue PM, Isaacs AJ, Wild SH \& Jacobs HS. Mortality of woman with polycystic ovary syndrome at long term follow up. Journal of Clinical Epidemiology 199851 581-586.

38 Legro RS. Polycystic ovary syndrome and cardiovascular disease: a premature association? Endocrine Reviews 200324 302-312.

39 Shaw LJ, Merz CN, Azziz R, Stanczyk FZ, Sopko G, Braunstein GD, Kelsey SF, Kip KE, Cooper-Dehoff RM, Johnson BD, Vaccarino V, Reis SE, Bittner V, Hodgson TK, Rogers W \& Pepine CJ. Postmenopausal women with a history of irregular menses and elevated androgen measurements at high risk for worsening cardiovascular event-free survival: results from the National Institutes of Health National Heart, Lung, and Blood Institute (NHLBI) Sponsored Women's Ischemia Syndrome Evaluation (WISE). Journal of Clinical Endocrinology and Metabolism 200893 $1276-1284$.

Received 24 June 2009

Accepted 14 July 2009 